\title{
Possible Cross-Section for a Coronal Loop of Given Shape?
}

\author{
M.S. Ruderman
}

Received: 12 August 2014 / Accepted: 3 October 2014 / Published online: 14 October 2014

(C) The Author(s) 2014. This article is published with open access at Springerlink.com

\begin{abstract}
We aim to answer the question about the cross-section of a planar coronal loop with a prescribed shape. We restrict the analysis to coronal loops embedded in a planar potential magnetic field. Then we carry out the analysis in the leading-order approximation with respect to the small parameter $\epsilon$ equal to the ratio of the characteristic size of the loop cross-section to the loop length. We show that, in this approximation, the loop cross-section can be prescribed arbitrarily at one of its footpoints. Then the loop cross-section at any other point is obtained by stretching or compressing the prescribed loop cross-section in the direction that is perpendicular to the loop axis and in the plane of the loop. The variation of the coefficient of stretching or compression along the loop can be chosen arbitrarily. In particular, it follows from this result that we can consider a planar loop of arbitrary shape and assume that its cross-section is circular everywhere and has a constant radius.
\end{abstract}

Keywords Sun $\cdot$ Plasma $\cdot$ Coronal loops $\cdot$ Magnetohydrodynamics $\cdot$ Magnetic equilibrium

\section{Introduction}

Standing kink waves in coronal magnetic loops were first observed by the Transition Region and Corona Explorer (TRACE) on 14 July 1998. These observations were reported by Aschwanden et al. (1999) and Nakariakov et al. (1999). Later, similar observations were reported by, e.g., Schrijver and Brown (2000), Aschwanden et al. (2002), Schrijver, Aschwanden, and Title (2002), Aschwanden (2006), and Aschwanden and Schrijver (2011).

Since the standing kink waves in coronal loops were first observed, they have attracted great attention from theorists. In the first theoretical works related to these waves, the simplest model of a coronal magnetic loop was used, which is a straight homogeneous magnetic

M.S. Ruderman (凶)

Solar Physics and Space Plasma Research Centre (SP2RC), School of Mathematics and Statistics, University of Sheffield, Hicks Building, Hounsfield Road, Sheffield, S3 7RH, UK

e-mail: m.s.ruderman@sheffield.ac.uk

M.S. Ruderman

Space Research Institute (IKI) Russian Academy of Sciences, Moscow, Russia 
tube. The theory of kink-wave propagation in straight homogeneous magnetic tubes was developed about two decades before they were first observed (e.g. Ryutov and Ryutova, 1976; Edwin and Roberts, 1983). Then more complicated models were developed with properties of coronal magnetic loops such as density variation along and across the loop, loop expansion, curvature, torsion, and magnetic twist. For a recent review of the theory of standing kink waves in coronal magnetic loops see Ruderman and Erdélyi (2009).

A very popular approximation used in the theory of kink waves in coronal loops is the thin-tube (TT) approximation. It is based on the fact that the size of the loop cross-section is much smaller than the loop length, so the ratio of these two quantities can be considered as a small parameter. The use of the TT approximation greatly simplifies the theory of kink waves in coronal loops. It was assumed in most studies that the loop cross-section is circular. It is also typical to neglect the loop curvature. In particular, Dymova and Ruderman (2005) showed that in the TT approximation, kink waves in a straight magnetic tube with a circular cross-section with constant radius and the density varying along the loop are described by a wave equation. Ruderman, Verth, and Erdélyi (2008) and Verth and Erdélyi (2008) generalized this result to include the cross-section radius variation along the loop.

At present, it is not possible to arrive at any conclusion about the shape of the loop crosssection, so it is not clear how relevant the model of a loop with a circular cross-section is. Ruderman (2003) and Morton and Ruderman (2011) studied kink oscillations of a loop with an elliptic cross-section. They found that the main effect of the cross-section ellipticity is that the frequencies of oscillations polarized along the small and large axis of the cross-section are different.

Van Doorsselaere et al. (2004) analytically and Terradas, Oliver, and Ballester (2006) numerically studied the effect of loop curvature on kink oscillations of coronal loops (see also the review by Van Doorsselaere, Verwichte, and Terradas, 2009). They showed that the effect of curvature is very small in the TT approximation and can be neglected. However, these authors assumed that the loop has a half-circle shape and the loop cross-section is a circle of constant radius. Ruderman (2009) studied kink oscillations of a planar curved loop of arbitrary shape embedded in a potential planar magnetic field. He showed that, in general, the loop cross-section is elliptic. The ellipse axis perpendicular to the loop plane is constant, while the second ellipse axis varies along the loop. As a result, the loop kink oscillation polarized in the direction perpendicular to the loop plane has a frequency different from that of the oscillation polarized in the loop plane.

The loop shape is important first of all because it determines the variation of the plasma density along the loop when the dependence of the temperature on the height in the corona is given. The density variation along the loop, in turn, affects the ratio of frequencies of the first overtone and fundamental mode of kink oscillations. This ratio is one of the most important parameters in coronal seismology because it is used to estimate the atmospheric scale height in the corona in the framework of the method developed by Andries, Arregui, and Goossens (2005) (see also the review by Andries et al., 2009).

In the seminal article by Andries, Arregui, and Goossens (2005), the loop was assumed to have a half-circle shape and a circular cross-section of constant radius. Dymova and Ruderman (2006) were the first to study the effect of the loop shape on the frequency ratio. These authors considered loops with the shape of an arc of a circle and a circular crosssection of constant radius, and they studied the dependence of the frequency ratio on the ratio of the loop height to the distance between the loop footpoints. Later, this study was continued by other authors. Morton and Erdélyi (2009) studied kink oscillations of a coronal loop with an elliptic shape and calculated the dependence of the frequency ratio on the ellipticity parameter. Orza and Ballai (2013) considered asymmetric loops and studied the 
dependence of the frequency ratio on the loop asymmetry. Both Morton and Erdélyi (2009) and Orza and Ballai (2013) assumed that the loop cross-section is a circle of constant radius.

It is very easy to obtain an equilibrium with a loop of half-circle shape and circular crosssection of constant radius embedded in a potential planar magnetic field. The same is true for a loop with an arc-of-circle shape. However, in view of the study by Ruderman (2009), it is not at all clear if it is possible to obtain an equilibrium with a planar loop of, for example, elliptic shape with a circular cross-section of constant radius embedded in a planar potential magnetic field. Moreover, it is not even clear whether a planar loop with an arbitrary shape can be embedded in a planar potential magnetic field. Hence, important questions arise: Can a loop with an arbitrary shape be embedded in a planar potential magnetic field? If the loop shape is given and the loop is embedded in a planar potential magnetic field, then what can be its cross-section? In particular, is it possible to have a loop with the circular cross-section of constant radius and with given shape?

This article aims to answer these questions. It is organized as follows: In the next section we formulate the problem. In Section 3 we obtain the solution describing an equilibrium with a planar magnetic tube embedded in a planar potential magnetic field and study the variation of the loop cross-section along the loop. Section 4 contains the summary and our conclusions.

\section{Problem Formulation}

We consider a planar potential magnetic field in the upper part of the $x-z$-plane defined by $z \geq 0$. The two components of the magnetic field can be expressed in terms of the magnetic flux function $[\psi]$ as

$$
B_{x}=\frac{\partial \psi}{\partial z}, \quad B_{z}=-\frac{\partial \psi}{\partial x},
$$

where $\psi$ satisfies the Laplace equation

$$
\frac{\partial^{2} \psi}{\partial x^{2}}+\frac{\partial^{2} \psi}{\partial z^{2}}=0
$$

We assume that the loop axis is defined by the parametric equations

$$
x=x_{0}(s), \quad z=z_{0}(s),
$$

where $z_{0}(0)=z_{0}(L)=0, L$ is the loop length, $s$ is the length counted along the loop axis, and $z_{0}(s) \geq 0$ for $0 \leq s \leq L$. We denote this curve as $\mathcal{C}$. Since $s$ is the distance along the loop, we have the relation

$$
x_{0}^{\prime 2}+z_{0}^{\prime 2}=1,
$$

where the prime indicates the derivative with respect to $s$. We introduce the size of the loop cross-section as the greatest distance between two points at the cross-section boundary. In what follows we assume that the ratio of the cross-section size to $L$ is low and does not exceed $\epsilon \ll 1$.

For simplicity, we assume below that $z_{0}(s)$ monotonically increases in the interval $\left(0, s_{a}\right)$ and monotonically decreases in the interval $\left(s_{a}, L\right)$, where $s_{a}$ corresponds to the loop apex. As for $x_{0}(s)$, we assume that it either monotonically increases in the whole interval $(0, L)$, or monotonically decreases in the interval $\left(0, s_{1}\right)$, and then monotonically increases in the interval $\left(s_{1}, L\right)$, or monotonically decreases in the interval $\left(0, s_{1}\right)$, then monotonically increases 

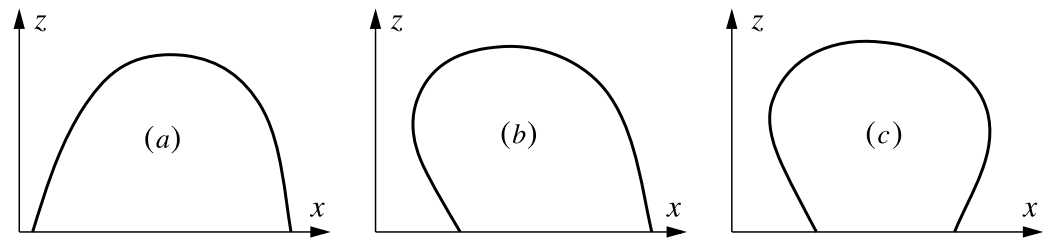

Figure 1 Three different loop shapes.

Figure 2 The local coordinate system. The thick line is the loop axis. The length $s$ along the loop is measured from the left footpoint. The coordinate $u$ is measured from the loop axis along a normal line to the loop axis.

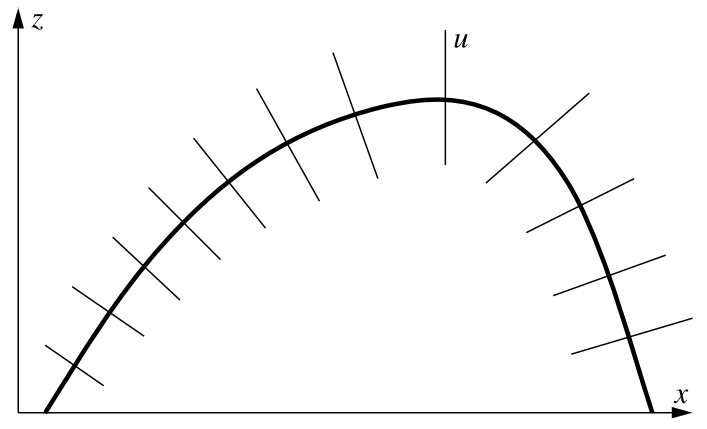

in the interval $\left(s_{1}, s_{2}\right)$, and then again monotonically decreases in the interval $\left(s_{2}, L\right)$, where $0<s_{1}<s_{a}<s_{2}<L$. We also assume that the loop axis is a concave curve. The three possible shapes of the loop are shown in Figures 1a, b, and c. Other assumptions are that the distance between the loop footpoints is of the order of $L$, and the smallest radius of the loop curvature is also of the order of $L$.

\section{Loop Cross-Section}

We start by introducing a local curvilinear coordinate system in the vicinity of the loop axis. To do this, we draw straight lines normal to the loop axis at each point (see Figure 2). We denote the length along a straight line measured from the point of its intersection with the loop axis as $u$. The quantity $u$ is negative when it is measured inside the region bounded by the loop axis and the $x$-axis, and positive when it is measured outside. This is a local coordinate system because the normal lines, in general, intersect at some distance from the loop axis. However, we only use this coordinate system in the vicinity of the loop axis defined by the condition $|u| \leq d$. We show in Appendix A that it is enough to impose the restriction that $d$ is smaller than half of the minimum curvature radius of the loop axis and also smaller than half of the distance between the loop footpoints. Hence, if we assume that these two quantities are much larger than $\epsilon L$, then we can take $d$ much larger than $\epsilon L$. The equation of the normal line crossing the loop axis at the point $\left(x_{0}, z_{0}\right)$ is

$$
x=x_{0}(s)-u z_{0}^{\prime}(s), \quad z=z_{0}(s)+u x_{0}^{\prime}(s) .
$$

These equations give the relation between the curvilinear coordinates $(s, u)$ and Cartesian coordinates $(x, z)$. Differentiating the identities $x \equiv x(s(x, z), u(x, z))$ and $z \equiv$ $z(s(x, z), u(x, z))$ with respect to $x$ and $z$ yields

$$
\frac{\partial s}{\partial x}=\frac{1}{\Delta} \frac{\partial z}{\partial u}, \quad \frac{\partial s}{\partial z}=-\frac{1}{\Delta} \frac{\partial x}{\partial u}, \quad \frac{\partial u}{\partial x}=-\frac{1}{\Delta} \frac{\partial z}{\partial s}, \quad \frac{\partial u}{\partial z}=\frac{1}{\Delta} \frac{\partial x}{\partial s},
$$


where

$$
\Delta=\frac{\partial x}{\partial s} \frac{\partial z}{\partial u}-\frac{\partial x}{\partial u} \frac{\partial z}{\partial s} .
$$

Using Equations (4) and (5), we obtain

$$
\Delta=1-\kappa u, \quad \kappa=x_{0}^{\prime} z_{0}^{\prime \prime}-x_{0}^{\prime \prime} z_{0}^{\prime},
$$

where $\kappa$ is the loop-axis curvature. The curvature $[\kappa]$ is positive if the tangent vector $\left(x_{0}^{\prime}, z_{0}^{\prime}\right)$ to the loop axis rotates counter-clockwise when $s$ increases and negative otherwise. It follows from the assumption that the loop axis is a concave curve that its curvature is everywhere negative.

With the aid of Equations (4), (5), and (8), we reduce Equation (6) to

$$
\begin{aligned}
\frac{\partial s}{\partial x} & =\frac{x_{0}^{\prime}}{1-\kappa u}, & \frac{\partial s}{\partial z} & =\frac{z_{0}^{\prime}}{1-\kappa u}, \\
\frac{\partial u}{\partial x} & =-\frac{z_{0}^{\prime}+u x_{0}^{\prime \prime}}{1-\kappa u}, & \frac{\partial u}{\partial z} & =\frac{x_{0}^{\prime}-u z_{0}^{\prime \prime}}{1-\kappa u} .
\end{aligned}
$$

Using Equation (8), we obtain

$$
\kappa^{2}=\left(x_{0}^{\prime} z_{0}^{\prime \prime}-x_{0}^{\prime \prime} z_{0}^{\prime}\right)^{2}=x_{0}^{\prime 2} z_{0}^{\prime \prime 2}-2 x_{0}^{\prime} z_{0}^{\prime} x_{0}^{\prime \prime} z_{0}^{\prime \prime}+x_{0}^{\prime \prime 2} z_{0}^{\prime 2} .
$$

Now we use Equation (4) to transform this equation to

$$
\kappa^{2}=x_{0}^{\prime \prime 2}+z_{0}^{\prime \prime 2}-x_{0}^{\prime 2} x_{0}^{\prime \prime 2}-2 x_{0}^{\prime} z_{0}^{\prime} x_{0}^{\prime \prime} z_{0}^{\prime \prime}-z_{0}^{\prime 2} z_{0}^{\prime \prime 2}=x_{0}^{\prime \prime 2}+z_{0}^{\prime \prime 2}-\left(x_{0}^{\prime} x_{0}^{\prime \prime}+z_{0}^{\prime} z_{0}^{\prime \prime}\right)^{2} .
$$

In accordance with Equation (4), the second term on the right-hand side of this equation is zero, so eventually we arrive at

$$
\kappa^{2}=x_{0}^{\prime \prime 2}+z_{0}^{\prime \prime 2} .
$$

Using Equations (4), (8), (9), and (12), we rewrite Equation (2) in terms of $s$ and $u$ as

$$
\frac{\partial}{\partial s}\left(\frac{1}{1-\kappa u} \frac{\partial \psi}{\partial s}\right)+\frac{\partial}{\partial u}\left((1-\kappa u) \frac{\partial \psi}{\partial u}\right)=0 .
$$

We look for the solution to this equation in the form of power series:

$$
\psi=\sum_{n=0}^{\infty} u^{n} \psi_{n}(s)
$$

In what follows we assume that the loop axis is a magnetic-field line. This implies that $\psi=$ const. when $u=0$. Without loss of generality, we can take $\psi=0$ when $u=0$. Then $\psi_{0}(s)=0$. Now, substituting Equation (14) in Equation (13) and collecting terms with the same power of $u$, we obtain the infinite set of equations for the coefficients $\psi_{n}(s)$. It is convenient to write the first two equations of this set separately. As a result, we have

$$
\begin{aligned}
2 \psi_{2}= & \kappa \psi_{1}, \\
6 \psi_{3}= & 8 \kappa \psi_{2}-\psi_{1}^{\prime \prime}-2 \kappa^{2} \psi_{1}, \\
(n+1)(n+2) \psi_{n+2}= & (n+1)(3 n+1) \kappa \psi_{n+1}-\psi_{n}^{\prime \prime}-n(3 n-1) \kappa^{2} \psi_{n} \\
& +\kappa \psi_{n-1}^{\prime \prime}-\kappa^{\prime} \psi_{n-1}^{\prime}-(n-1)^{2} \kappa^{3} \psi_{n-1}, \quad n=2,3, \ldots
\end{aligned}
$$

We can see that we can take $\psi_{1}(s)$ arbitrarily and then calculate $\psi_{n}(s), n=2,3$, etc., using Equations (15)-(17). It is proved in Appendix B that the series (14) is convergent if $x_{0}(s)$, 
$y_{0}(s)$, and $\psi_{1}(s)$ are sufficiently "good" functions, i.e. they satisfy all restrictions imposed in Appendix B, and $d$ is sufficiently small, but still much larger than $\epsilon L$.

In what follows we use only the first non-zero term in the series in Equation (14) and assume that $|u| \lesssim \epsilon L$, so

$$
\psi=u \psi_{1}(s)[1+\mathcal{O}(\epsilon)] .
$$

Consider the plane $[\Pi]$ orthogonal to the loop axis at its left footpoint. Since the vector $\boldsymbol{e}_{u}=\left(-z_{0}^{\prime}(0), x_{0}^{\prime}(0)\right)$ is orthogonal to the loop axis at this footpoint $\left[\boldsymbol{e}_{u} \in \Pi\right]$. We denote the unit vector in the $y$-direction, which is the direction orthogonal to the plane of the loop, as $\boldsymbol{e}_{y}$. Since $\boldsymbol{e}_{u} \perp \boldsymbol{e}_{y}$, these two vectors can be considered as the unit vector of Cartesian coordinates in the plane [П]. The corresponding coordinates are $u$ and $y$, and the coordinate origin coincides with the intersection of $\Pi$ and the loop axis.

Now we consider a closed contour $\Gamma \in \Pi$. It is defined by the equations

$$
u=u_{0}(t), \quad y=y_{0}(t),
$$

where $u_{0}(T)=u_{0}(0)$ and $y_{0}(T)=y_{0}(0)$. We assume that $\Gamma$ is a simple contour, i.e. it does not have self-intersections, and the coordinate origin is inside $\Gamma$. In addition, we assume that $\Gamma$ is convex, i.e. any straight line can intersect it at no more than two points. The numbers $u, y$, and $s$ define orthogonal curvilinear coordinates in three-dimensional space. In these coordinates the equation of the loop axis is $u=y=0$. The magnetic line crossing $\Gamma$ at a point with coordinates $\left(u_{0}(t), y_{0}(t)\right)$ is defined by the equations $y=y_{0}(t)$ and $\psi(u, s)=$ $\psi\left(u_{0}(t), 0\right)$. Varying $s$ from 0 to $L$, we obtain a part of this line bounded by the two planes orthogonal to the loop axis at the footpoints. Now we also vary $t$ from 0 to $T$. Then we obtain a magnetic surface consisting of magnetic lines crossing the contour $\Gamma$. We can consider this surface as the surface of the magnetic tube. Then, using Equation (18), we write the equation of the surface of the magnetic tube as

$$
y=y_{0}(t), \quad u \psi_{1}(s)=u_{0}(t) \psi_{1}(0)[1+\mathcal{O}(\epsilon)], \quad 0 \leq s \leq L, 0 \leq t \leq T .
$$

The boundary of the tube cross-section by the plane perpendicular to the tube axis at $s=\bar{s}$ is defined by the equation

$$
y=y_{0}(t), \quad u=u_{0}(t) \frac{\psi_{1}(0)}{\psi_{1}(\bar{s})}[1+\mathcal{O}(\epsilon)], \quad 0 \leq t \leq T .
$$

We see that, in the leading-order approximation with respect to $\epsilon$, the tube cross-section at $s=\bar{s}$ is obtained from the tube cross-section at $s=0$ by stretching or compressing this latter cross-section in the $u$-direction with the coefficient $\psi_{1}(0) / \psi_{1}(\bar{s})$. In particular, if we take $\Gamma$ to be a circle of radius $a$ centred at the loop axis, and we also take $\psi_{1}(s) \equiv 1$, then we obtain that in the leading-order approximation with respect to $\epsilon$, the tube cross-section is the circle of radius $a$ centred at the loop axis everywhere. Hence, we can consider a planar loop of arbitrary shape and assume that it has a circular cross-section with constant radius everywhere.

\section{Summary and Conclusions}

We have answered the question about the kind of cross-section a planar coronal loop with a prescribed shape can have. This question arises in relation to works such as those of Morton and Erdélyi (2009) and Orza and Ballai (2013), where the authors considered kink oscillations of curved planar loops with prescribed shapes and assumed that the loop cross-section is circular everywhere and has a constant radius. 
In our analysis we assumed that the loop is embedded in a planar potential magnetic field. We also assumed that the loop axis is described by an analytical function. We showed that the loop cross-section can be prescribed arbitrarily at one of the loop footpoints. Then the loop cross-section at any other point is obtained by stretching or compressing the prescribed loop cross-section in the direction that is perpendicular to the loop axis and in the plane of the loop. The variation of the coefficient of stretching/compression along the loop can be chosen arbitrarily. Note that this result is only valid in the leading-order approximation with respect to the small parameter $\epsilon$, which is the ratio of the characteristic size of the loop cross-section to the loop length.

In particular, it follows from the result we obtained that we can consider a planar loop of arbitrary shape and assume that its cross-section is circular everywhere and has a constant radius.

Acknowledgements The author acknowledges the support by an STFC grant (R/131168), and by the Russian Fund for Fundamental Research (RFBR) grant (13-02-00656).

Open Access This article is distributed under the terms of the Creative Commons Attribution License which permits any use, distribution, and reproduction in any medium, provided the original author(s) and the source are credited.

\section{Appendix A: Proof That the Map $(s, u) \rightarrow(x, z)$ Is Bijective}

In this section we consider the map $(s, u) \rightarrow(x, z)$ defined by Equation (5). We slightly extend the curve $\mathcal{C}$ beyond the footpoints. This can be done in infinitely many ways. For example, we can use small arcs of circles of radii equal to the curvature radii at points $\left(x_{0}(0), 0\right)$ and $\left(x_{0}(L), 0\right)$ (see Figure 3 ). These circles are centred at the centres of curvature. The arc lengths are equal to $\delta \ll L$. We denote the extended curve as $\widetilde{\mathcal{C}}$. Note that this curve is not only smooth, but it also has a continuous curvature. The lines normal to $\widetilde{\mathcal{C}}$ at its end points have equations

$$
\begin{aligned}
& x=x_{0}(-\delta)-u z_{0}^{\prime}(-\delta), \quad z=z_{0}(-\delta)+u x_{0}^{\prime}(-\delta), \\
& x=x_{0}(L+\delta)-u z_{0}^{\prime}(L+\delta), \quad z=z_{0}(L+\delta)+u x_{0}^{\prime}(L+\delta) .
\end{aligned}
$$

We denote the first line as $\ell_{-}$and the second as $\ell_{+}$. Now we introduce two curves $\widetilde{\mathcal{C}}_{-}$and $\widetilde{\mathcal{C}_{+}}$defined by the equations

$$
\begin{array}{lll}
x=x_{0}(s)+d z_{0}^{\prime}(s), & z=z_{0}(s)-d x_{0}^{\prime}(s), & s \in[-\delta, L+\delta], \\
x=x_{0}(s)-d z_{0}^{\prime}(s), & z=z_{0}(s)+d x_{0}^{\prime}(s), & s \in[-\delta, L+\delta],
\end{array}
$$

respectively, where $d$ is sufficiently small. The exact restriction on $d$ will be imposed later. Consider the rectangle $\mathcal{D}$ in the plane $(s, u)$ defined by $-\delta<s<L+\delta,|u|<d$, and the domain $\mathcal{R}$ bounded by the lines $\ell_{-}$and $\ell_{+}$, and by the curves in $\widetilde{\mathcal{C}}_{-}$and $\widetilde{\mathcal{C}_{+}}$in the plane $(x, z)$. Equation (5) defines a map $F: \mathcal{D} \rightarrow \mathcal{R}$.

First we show that the map $F$ is injective. This means that $F\left(s_{a}, u_{a}\right) \neq F\left(s_{b}, u_{b}\right)$ if $\left(s_{b}, u_{b}\right) \neq\left(s_{a}, u_{a}\right)$. We assume the contrary, that there are two points, $\left(s_{a}, u_{a}\right) \in \mathcal{D}$ and $\left(s_{b}, u_{b}\right) \in \mathcal{D}$, such that $F\left(s_{a}, u_{a}\right)=F\left(s_{b}, u_{b}\right)=(x, z)$. This implies that the two normal lines to the curve $\widetilde{\mathcal{C}}$, one at point $\mathrm{A}$ and the other at point $\mathrm{B}$, intersect at point $\mathrm{C} \in \mathcal{R}$, where $\mathrm{A}$ and $\mathrm{B}$ are the points on the curve $\widetilde{\mathcal{C}}$ corresponding to $s=s_{a}$ and $s=s_{b}$. Since $\mathrm{C} \in \mathcal{R}$, we have $|\mathrm{AC}|=d_{a}<d$ and $|\mathrm{BC}|=d_{b}<d$. We introduce the angle $[\theta]$ between the tangent 
Figure 3 Sketch of the domain $\mathcal{R}$.

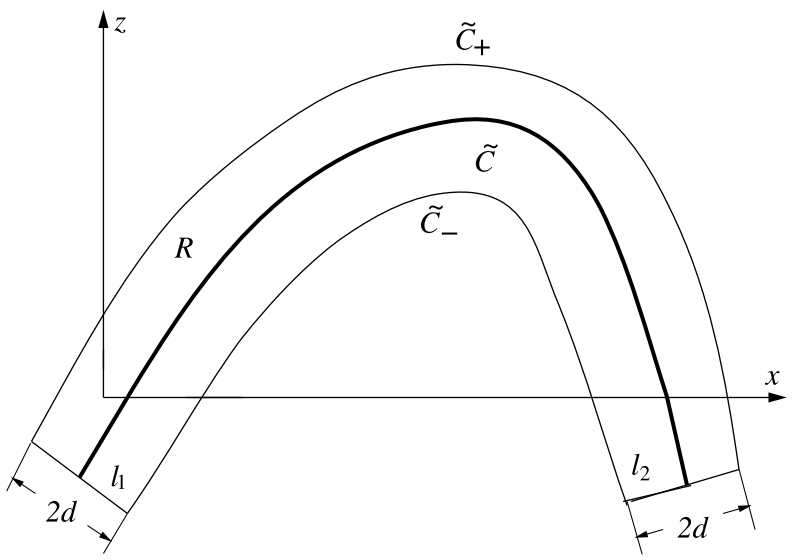

Figure 4 Illustration for the proof that the map $F$ is injective.

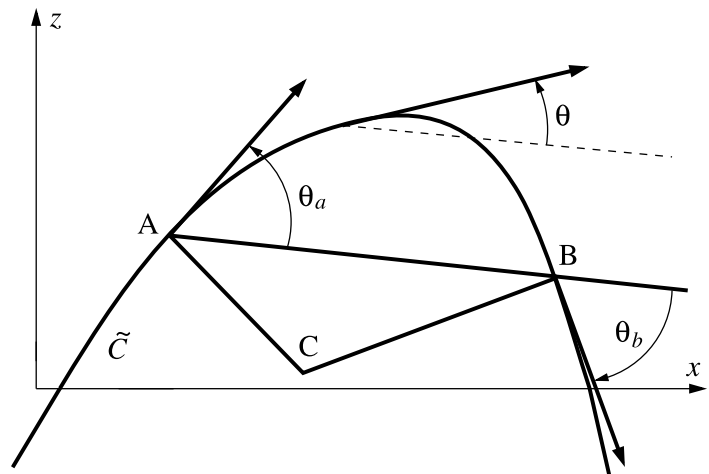

vector to $\widetilde{\mathcal{C}}$ and the line $\mathrm{AB}$ connecting the points $\mathrm{A}$ and $\mathrm{B}$ (see Figure 4). This angle takes the values $\theta_{a}$ and $\theta_{b}$ at points $\mathrm{A}$ and $\mathrm{B}$. The distance between the points $\mathrm{A}$ and $\mathrm{B}$ is given by

$$
|\mathrm{AB}|=\int_{s_{a}}^{s_{b}} \cos \theta \mathrm{d} s
$$

Using the relation $\mathrm{d} \theta / \mathrm{d} s=\kappa(s)$, we transform this expression to

$$
|\mathrm{AB}|=\int_{\theta_{a}}^{\theta_{b}} \frac{\cos \theta}{\kappa} \mathrm{d} \theta .
$$

Let $\kappa_{m}=\max |\kappa|$ for $s \in[-\delta, L+\delta]$. Taking into account that $-\kappa_{m} \leq \kappa \leq 0$ and $\theta_{b}<0$, we obtain from Equation (27) the estimate

$$
|\mathrm{AB}| \geq \frac{\sin \theta_{a}+\sin \left|\theta_{b}\right|}{\kappa_{m}}=\frac{2}{\kappa_{m}} \sin \frac{\theta_{a}+\left|\theta_{b}\right|}{2} \cos \frac{\theta_{a}-\left|\theta_{b}\right|}{2} .
$$

It is straightforward to see that $\angle \mathrm{BAC}=\pi / 2-\theta_{a}, \angle \mathrm{ABC}=\pi / 2-\left|\theta_{b}\right|$, and, consequently, $\angle \mathrm{ACB}=\theta_{a}+\left|\theta_{b}\right|$. Then, using the law of sines, we obtain

$$
\frac{\cos \theta_{a}}{|\mathrm{BC}|}=\frac{\cos \theta_{b}}{|\mathrm{AC}|}=\frac{\sin \left(\theta_{a}+\left|\theta_{b}\right|\right)}{|\mathrm{AB}|} .
$$


Figure 5 Illustration of the proof that the map $F$ is injective for the case when the loop axis has the shape shown in Figure 1b and the angle between the normals is larger than $\pi$.

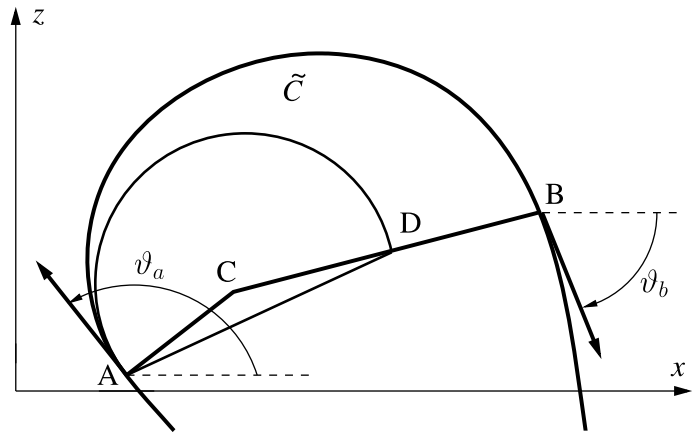

Now we impose the restriction that $d<1 / 2 \kappa_{m}$. Since $|\mathrm{AC}|<d<1 / 2 \kappa_{m}$ and $|\mathrm{BC}|<d<$ $1 / 2 \kappa_{m}$, it follows from Equation (29) that

$$
|\mathrm{AB}|<\frac{\sin \left(\theta_{a}+\left|\theta_{b}\right|\right)}{\kappa_{m}\left(\cos \theta_{a}+\cos \theta_{b}\right)}=\frac{\sin \frac{\theta_{a}+\left|\theta_{b}\right|}{2}}{\kappa_{m} \cos \frac{\theta_{a}-\left|\theta_{b}\right|}{2}} .
$$

It follows from Equations (28) and (30) that $2 \cos \left[\left(\theta_{a}-\left|\theta_{b}\right|\right) / 2\right]<1$ and, consequently,

$$
\cos \left(\theta_{a}-\left|\theta_{b}\right|\right)<0 \text {. }
$$

Since $0<\theta_{a} \leq \pi / 2$ and $0<\left|\theta_{b}\right| \leq \pi / 2$, the inequality in Equation (31) cannot be satisfied, and we arrive at the contradiction. Hence, the normal lines at $\mathrm{A}$ and $\mathrm{B}$ cannot intersect in $\mathcal{R}$.

It is obvious that the proof presented above is only valid when the angle $\angle \mathrm{ACB}$ is smaller than $\pi$. This is always true when the loop axis has the shape shown in Figure 1a. However, when it has either the shape shown in Figure 1b or that shown in Figure 1c, it is also possible that $\angle \mathrm{ACB}$ is larger than $\pi$. We consider the case shown in Figure $1 \mathrm{~b}$ and assume that the angle between the normal lines at $\mathrm{A}$ and $\mathrm{B}$ counted from the second line counter-clockwise is larger than $\pi$ (see Figure 5). Let $\vartheta$ be the angle between the tangent to $\widetilde{\mathcal{C}}$ and the positive $x$-axis counted counter-clockwise from the $x$-axis. For the case shown in Figure $1 \mathrm{~b}, \pi / 2<$ $\vartheta_{a}<\pi$ and $-\pi / 2 \leq \vartheta_{b} \leq 0$, so $\angle \mathrm{ACB}=\vartheta_{a}-\vartheta_{b}<3 \pi / 2$. We draw the arc AD of the circle of radius $1 / \kappa_{m}$ that touches the curve $\widetilde{\mathcal{C}}$ at $\mathrm{A}$. This arc cannot cross $\widetilde{\mathcal{C}}$ because otherwise the absolute curvature of $\widetilde{\mathcal{C}}$ would have to exceed $\kappa_{m}$ somewhere between $\mathrm{A}$ and the point of intersection. The second end of this arc cannot be on AC because, by assumption, $|\mathrm{AC}|$ is smaller than the half of the curvature radius. Since $2 \pi-\angle \mathrm{ACB}>2 \pi-3 \pi / 2=\pi / 2$, it follows that $|\mathrm{AD}|>\left(2 / \kappa_{m}\right) \sin (\pi / 4)=\sqrt{2} / \kappa_{m}$. Now we have

$$
|\mathrm{AC}|+|\mathrm{BC}| \geq|\mathrm{AC}|+|\mathrm{CD}|>|\mathrm{AD}|>\frac{\sqrt{2}}{\kappa_{m}},
$$

which again contradicts the assumption that $\mathrm{C} \in \mathcal{R}$ because, in that case, we would have $|\mathrm{AC}|+|\mathrm{BC}|<2 d<1 / \kappa_{m}$.

Finally, we consider a loop with the axis of the shape in Figure 1c and assume that the angle between the normals is larger than $\pi$ (see Figure 6). At points $\mathrm{D}$ and $\mathrm{E}$ the tangents to the curve $\widetilde{\mathcal{C}}$ are vertical. It is straightforward to see that if $A$ is above $D$, as in Figure 6 a, or B is above E, as in Figure 6b, then the proof is the same as for a loop with the axis of shape (b). Therefore we only need to consider the case where both $\mathrm{A}$ is below $\mathrm{D}$ and $\mathrm{B}$ is below $\mathrm{E}$. Then it is obvious that $|\mathrm{AC}|+|\mathrm{BC}|$ is larger than the distance between the end points of the curve $\widetilde{\mathcal{C}}$. We introduce $d_{f}=x_{0}(L)-x_{0}(0)$. Then we have for the distance between the end points of the curve $\widetilde{\mathcal{C}}$

$$
x_{0}(L+\delta)-x_{0}(-\delta)>d_{f}-2 \delta
$$



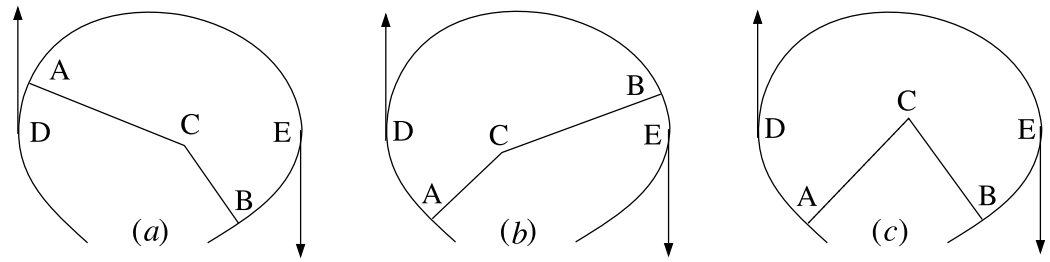

Figure 6 Illustration of the proof that the map $F$ is injective and has the shape shown in Figure $1 \mathrm{c}$ and the angle between the normals is larger than $\pi$.

Now we impose the addition restriction $d<d_{f} / 2-\delta$. Then we have that on the one hand $|\mathrm{AC}|+|\mathrm{BC}|>d_{f}-2 \delta$, but on the other hand $|\mathrm{AC}|+|\mathrm{BC}|<2 d<d_{f}-2 \delta$, and again we arrive at the contradiction.

Hence, to summarize, we have proved that the map $F$ is injective if $d<\min \left(1 / 2 \kappa_{m}, d_{f} /\right.$ $2-\delta)$. To finish the proof that $F$ is bijective, we now need to prove that for any point $(x, z) \in \mathcal{R}$ there is a point $(s, u) \in \mathcal{D}$ such that $F(s, u)=(x, z)$.

First of all, we note that $F$ can be extended on the closure $\overline{\mathcal{D}}$ of $\mathcal{D}$. Then it is straightforward to see that $F$ is a bijection of the boundary of $\mathcal{D}$ on the boundary of $\mathcal{R}$. Now we assume that there is point $\mathrm{A} \in \mathcal{R}$ such that no point from $\mathcal{D}$ is mapped in $\mathrm{A}$. There are two possibilities: Either there is a point in any vicinity of A that is the image of a point from $\mathcal{D}$, or there is a vicinity of A such that no point in this vicinity is the image of a point from $\mathcal{D}$.

Consider the first possibility. In this case, we can construct a sequence of points $\left\{\mathrm{A}_{n}\right\}$ converging to $\mathrm{A}$ and such that, for each point $\mathrm{A}_{n}$, there is point $\mathrm{B}_{n} \in \mathcal{D}$ such that $F\left(\mathrm{~B}_{n}\right)=\mathrm{A}_{n}$. Since $\left\{\mathrm{A}_{n}\right\}$ is converging and $|\Delta|$ is bounded from below by a positive constant, it follows that $\left\{\mathrm{B}_{n}\right\}$ is a Cauchy sequence and, consequently, it converges to a point $\mathrm{B}$. Since $F$ is continuous, it follows that $F(\mathrm{~B})=\mathrm{A}$, and we arrive at a contradiction. Note that $\mathrm{B}$ is not at the boundary of $\mathcal{D}$ because the boundary of $\mathcal{D}$ is mapped into the boundary of $\mathcal{R}$.

Assume now that there is a vicinity $[\mathcal{V}]$ of A such that no point in this vicinity is an image of a point from $\mathcal{D}$. We consider a simple closed contour $[\mathcal{L}]$ enclosing $\mathcal{V}$ and such that each point of this contour is the image of a point from $\mathcal{D}$. Such a contour definitely exists. For example, we can take the boundary of $\mathcal{R}$. The contour $[\mathcal{L}]$ is the image of the contour $\mathcal{S} \in \mathcal{D}$, $F(\mathcal{S})=\mathcal{L}$. Now we contract $\mathcal{S}$ into a point. Since $F$ is continuous, $\mathcal{L}$ also has to contract into a point. During this process, a part of $\mathcal{L}$ must be in $\mathcal{V}$. But this is impossible because, by assumption, no point in $\mathcal{V}$ is an image of a point from $\mathcal{D}$. Hence, we again arrive at a contradiction. As a result, we conclude that any point in $\mathcal{R}$ is an image of a point from $\mathcal{D}$, which means that $F$ is bijective.

\section{Appendix B: Investigation of the Sum Convergence}

In this appendix we study the convergence of the series in Equation (14). We assume that the functions $x_{0}(s), y_{0}(s)$, and $\psi(s)$ satisfy the inequalities

$$
\left|x_{0}^{(m)}(s)\right|<L h_{1}^{-m}, \quad\left|y_{0}^{(m)}(s)\right|<L h_{1}^{-m}, \quad\left|\psi_{1}^{(m)}(s)\right|<C_{2} h_{2}^{-m},
$$

where $h_{1}$ and $h_{2}$ are positive constants much larger than $\epsilon L, C_{2}$ is a positive constant, $m=0,1$, etc., and $f^{(m)}(s)$ denotes the $m$ th derivative of function $f(s)$. First we assume that these inequalities are valid for $s \in[0, L]$. The first two inequalities in Equation (34) imply that $x_{0}(s)$ and $y_{0}(s)$ are analytic functions in the interval $[0, L]$ and the radius of convergence of the infinite Taylor series of these functions at any point of this interval is 
larger than or equal to $h_{1}$. At the end point of the interval we have to take $s \geq 0$ and $s \leq L$ in these Taylor series. In Appendix A we described one possible extension of functions $x_{0}(s)$ and $y_{0}(s)$ using the arcs of circles. With the use of this extension we obtained the curve $\widetilde{\mathcal{C}}$ that is smooth and curves continuously. In this section we use another extension of curve $\mathcal{C}$. Using the expansion of $x_{0}(s)$ and $y_{0}(s)$ in the infinite Taylor series at the end points of the interval $[0, L]$, we can define them in the vicinities of points $s=0$ and $s=L$. As a result, we obtain functions $x_{0}(s)$ and $y_{0}(s)$ that are analytic in the interval $(-\delta, L+\delta)$ for sufficiently small positive $\delta$. Hence, we obtain the analytic curve $\widetilde{\mathcal{C}}$.

It follows from the inequalities in Equation (34) that we can find such positive constants $h$ and $C, h$ being much larger than $\epsilon L$, that

$$
\left|\kappa^{(m)}\right|<h^{-m-1}, \quad\left|\psi_{1}^{(m)}\right|<C h^{-m}, \quad m=0,1, \ldots
$$

Now we prove that

$$
\left|\psi_{n}^{(m)}\right|<C(n / h)^{m}(5 / h)^{n-1}, \quad n=2,3, \ldots, m=0,1, \ldots
$$

It follows from Equation (15) that

$$
2 \psi_{2}^{(m)}=\sum_{l=0}^{m}\left(\begin{array}{c}
m \\
l
\end{array}\right) \kappa^{(l)} \psi_{1}^{(m-l)},
$$

where $\left(\begin{array}{c}m \\ l\end{array}\right)$ is a binomial coefficient. Using Equation (35), we obtain from Equation (37)

$$
\left|\psi_{2}^{(m)}\right| \leq \frac{1}{2} \sum_{l=0}^{m}\left(\begin{array}{c}
m \\
l
\end{array}\right)\left|\kappa^{(l)}\right|\left|\psi_{1}^{(m-l)}\right|<\frac{C}{2 h^{m+1}} \sum_{l=0}^{m}\left(\begin{array}{c}
m \\
l
\end{array}\right)=\frac{2^{m} C}{2 h^{m+1}}<\frac{5 C}{h}\left(\frac{2}{h}\right)^{m} .
$$

Hence, the inequality (36) is valid for $n=2$.

In what follows we need estimates for the derivatives of $\kappa^{2}$ and $\kappa^{3}$. We have

$$
\left(\kappa^{2}\right)^{(m)}=\sum_{l=0}^{m}\left(\begin{array}{c}
m \\
l
\end{array}\right) \kappa^{(l)} \kappa^{(m-l)}
$$

It follows from this equation and Equation (35) that

$$
\left|\left(\kappa^{2}\right)^{(m)}\right| \leq \sum_{l=0}^{m}\left(\begin{array}{c}
m \\
l
\end{array}\right)\left|\kappa^{(l)}\right|\left|\kappa^{(m-l)}\right|<h^{-m-2} \sum_{l=0}^{m}\left(\begin{array}{c}
m \\
l
\end{array}\right)=2^{m} h^{-m-2} .
$$

Hence, eventually,

$$
\left|\left(\kappa^{2}\right)^{(m)}\right|<\frac{1}{4}(2 / h)^{m+2}
$$

Continuing, we obtain

$$
\left(\kappa^{3}\right)^{(m)}=\sum_{l=0}^{m}\left(\begin{array}{c}
m \\
l
\end{array}\right)\left(\kappa^{2}\right)^{(l)} \kappa^{(m-l)} .
$$

Now using Equations (35) and (42) yields

$$
\begin{aligned}
\left|\left(\kappa^{3}\right)^{(m)}\right| & \leq \sum_{l=0}^{m}\left(\begin{array}{c}
m \\
l
\end{array}\right)\left|\left(\kappa^{2}\right)^{(l)}\right|\left|\kappa^{(m-l)}\right|<\frac{1}{4} \sum_{l=0}^{m}\left(\begin{array}{c}
m \\
l
\end{array}\right)\left(\frac{2}{h}\right)^{l+2} h^{l-m-1} \\
& =h^{-m-3} \sum_{l=0}^{m}\left(\begin{array}{c}
m \\
l
\end{array}\right) 2^{l}=3^{m} h^{-m-3} .
\end{aligned}
$$


Hence, eventually we obtain

$$
\left|\left(\kappa^{3}\right)^{(m)}\right|<\frac{1}{27}(3 / h)^{m+3} .
$$

Differentiating Equations (16) yields

$$
6 \psi_{3}^{(m)}=8 \sum_{l=0}^{m}\left(\begin{array}{c}
m \\
l
\end{array}\right) \kappa^{(l)} \psi_{2}^{(m-l)}-\psi_{1}^{(m+2)}-2 \sum_{l=0}^{m}\left(\begin{array}{c}
m \\
l
\end{array}\right)\left(\kappa^{2}\right)^{(l)} \psi_{1}^{(m-l)} .
$$

Then, using Equations (35), (41), and (44), and Equation (36) with $n=2$, we obtain

$$
\begin{aligned}
\left|\psi_{3}^{(m)}\right| & \leq \sum_{l=0}^{m}\left(\begin{array}{c}
m \\
l
\end{array}\right)\left(\frac{4}{3}\left|\kappa^{(l)}\right|\left|\psi_{2}^{(m-l)}\right|+\frac{1}{3}\left|\left(\kappa^{2}\right)^{(l)}\right|\left|\psi_{1}^{(m-l)}\right|\right)+\frac{1}{6}\left|\psi_{1}^{(m+2)}\right| \\
& <\frac{C}{h^{m+2}}\left[\sum_{l=0}^{m}\left(\begin{array}{c}
m \\
l
\end{array}\right)\left(\frac{20}{3} 2^{m-l}+\frac{1}{3} 2^{l}\right)+\frac{1}{6}\right] \\
& =\frac{C}{h^{m+2}}\left(7 \cdot 3^{m}+\frac{1}{6}\right)<C\left(\frac{3}{h}\right)^{m}\left(\frac{5}{h}\right)^{2} .
\end{aligned}
$$

Hence, we see that the inequality (36) is valid for $n=3$. Now we use the method of mathematical induction. Assume that the inequalities (36) are valid for $2 \leq n \leq k$, where $k \geq 3$, and prove that they are then also valid for $n=k+1$. It follows from Equation (17) with $n=k-1$ that

$$
\begin{aligned}
k(k+1) \psi_{k+1}= & k(3 k-2) \kappa \psi_{k}-\psi_{k-1}^{\prime \prime}-(k-1)(3 k-4) \kappa^{2} \psi_{k-1} \\
& +\kappa \psi_{k-2}^{\prime \prime}-\kappa^{\prime} \psi_{k-2}^{\prime}-(k-2)^{2} \kappa^{3} \psi_{k-2} .
\end{aligned}
$$

Differentiating this equation yields

$$
\begin{aligned}
k(k+1) \psi_{k+1}^{(m)}= & \sum_{l=0}^{m}\left(\begin{array}{c}
m \\
l
\end{array}\right)\left[k(3 k-2) \kappa^{(l)} \psi_{k}^{(m-l)}+\kappa^{(l)} \psi_{k-2}^{(m-l+2)}\right. \\
& -(k-1)(3 k-4)\left(\kappa^{2}\right)^{(l)} \psi_{k-1}^{(m-l)}-\kappa^{(l+1)} \psi_{k-2}^{(m-l+1)} \\
& \left.-(k-2)^{2}\left(\kappa^{3}\right)^{(l)} \psi_{k-2}^{(m-l+1)}\right]-\psi_{k-1}^{(m+2)} .
\end{aligned}
$$

It follows from Equations (35), (41), (44), (48), and the assumption that the inequalities (36) are valid for $n \leq k$ that

$$
\begin{aligned}
k(k+1)\left|\psi_{k+1}^{(m)}\right| \leq & \sum_{l=0}^{m}\left(\begin{array}{c}
m \\
l
\end{array}\right)\left[k(3 k-2)\left|\kappa^{(l)}\right|\left|\psi_{k}^{(m-l)}\right|+\left|\kappa^{(l)}\right|\left|\psi_{k-2}^{(m-l+2)}\right|\right. \\
& +(k-1)(3 k-4)\left|\left(\kappa^{2}\right)^{(l)}\right|\left|\psi_{k-1}^{(m-l)}\right|+\left|\kappa^{(l+1)}\right|\left|\psi_{k-2}^{(m-l+1)}\right| \\
& \left.+(k-2)^{2}\left|\left(\kappa^{3}\right)^{(l)}\right|\left|\psi_{k-2}^{(m-l)}\right|\right]+\left|\psi_{k-1}^{(m+2)}\right| \\
< & \frac{C}{h^{m+k}} \sum_{l=0}^{m}\left(\begin{array}{c}
m \\
l
\end{array}\right)\left[k(3 k-2) 5^{k-1} k^{m-l}+(k-2)^{2} 5^{k-3}(k-2)^{m-l}\right. \\
& +(k-1)(3 k-4) 5^{k-2} 2^{l}(k-1)^{m-l}+(k-2) 5^{k-3}(k-2)^{m-l} \\
& \left.+(k-2)^{2} 5^{k-3} 3^{l}(k-2)^{m-l}\right]+C(k-1)^{m+2} 5^{k-2} h^{-m-k} \\
= & \frac{C}{h^{m}}\left(\frac{5}{h}\right)^{k}\left\{(k+1)^{m}\left[\frac{k(3 k-2)}{5}+\frac{(k-1)(4 k-5)}{25}+\frac{2(k-2)^{2}}{125}\right]\right.
\end{aligned}
$$




$$
\begin{aligned}
& \left.+(k-1)^{m}\left[\frac{(k-1)^{2}}{25}+\frac{(k-2)^{2}+k-2}{125}\right]\right\} \\
< & C\left(\frac{k+1}{h}\right)^{m}\left(\frac{5}{h}\right)^{k} \frac{97 k^{2}-96 k+40}{125}<k(k+1) C\left(\frac{k+1}{h}\right)^{m}\left(\frac{5}{h}\right)^{k} .
\end{aligned}
$$

Hence, we have proved that the inequalities (36) are valid for $n \leq k+1$. In accordance with the mathematical induction, this means that they are valid for any $n$. It follows from the inequality (36) that the series in Equation (14) as well as any series obtained from this series by term-by-term differentiating $m$ times with respect to $s$ and $l$ times with respect to $u$, where $m$ and $l$ are any non-negative integer numbers, are convergent with the radius of convergence larger than or equal to $h / 5$. Since $h$ is of the order of $L$, this radius of convergence is much larger than $\epsilon L$.

\section{References}

Andries, J., Arregui, I., Goossens, M.: 2005, Astrophys. J. Lett. 624, L57. DOI.

Andries, J., Van Doorsselaere, T., Roberts, B., Verth, G., Erdélyi, R.: 2009, Space Sci. Rev. 149, 3. DOI.

Aschwanden, M.J.: 2006, Phil. Trans. Roy. Soc. A 364, 417. DOI.

Aschwanden, M.J., Schrijver, C.J.: 2011, Astrophys. J. 736, 102. DOI.

Aschwanden, M.J., Fletcher, L., Schrijver, C.J., Alexander, D.: 1999, Astrophys. J. 520, 880. DOI.

Aschwanden, M.J., De Pontieu, B., Schrijver, C.J., Title, A.M.: 2002, Solar Phys. 206, 99. DOI.

Dymova, M., Ruderman, M.S.: 2005, Solar Phys. 229, 79. ADS. DOI.

Dymova, M., Ruderman, M.S.: 2006, Astron. Astrophys. 459, 241. DOI.

Edwin, P.M., Roberts, B.: 1983, Solar Phys. 88, 179. DOI.

Morton, R.J., Erdélyi, R.: 2009, Astron. Astrophys. 502, 315. DOI.

Morton, R.J., Ruderman, M.S.: 2011, Astron. Astrophys. 527, A53. DOI.

Nakariakov, V., Ofman, L., DeLuca, E.E., Roberts, B., Davila, J.M.: 1999, Science 285, 862. DOI.

Orza, B., Ballai, I.: 2013, Astron. Nachr. 334, 948. DOI.

Ruderman, M.S.: 2003, Astron. Astrophys. 409, 287. DOI.

Ruderman, M.S.: 2009, Astron. Astrophys. 506, 885. DOI.

Ruderman, M.S., Erdélyi, R.: 2009, Space Sci. Rev. 149, 199. DOI.

Ruderman, M.S., Verth, G., Erdélyi, R.: 2008, Astrophys. J. 686, 694. DOI.

Ryutov, D.D., Ryutova, M.P.: 1976, Sov. Phys. JETP 43, 491. ADS.

Schrijver, C.J., Brown, D.S.: 2000, Astrophys. J. Lett. 537, L69. DOI.

Schrijver, C.J., Aschwanden, M.J., Title, A.M.: 2002, Solar Phys. 206, 69. DOI.

Terradas, J., Oliver, R., Ballester, J.L.: 2006, Astrophys. J. Lett. 650, L91. DOI.

Van Doorsselaere, T., Debosscher, A., Andries, J., Poedts, S.: 2004, Astron. Astrophys. 424, 1065. DOI.

Van Doorsselaere, T., Verwichte, E., Terradas, J.: 2009, Space Sci. Rev. 149, 299. DOI.

Verth, G., Erdélyi, R.: 2008, Astron. Astrophys. 486, 1015. DOI. 